\title{
MODELOS DE VOLATILIDADE ESTOCÁSTICA EM SÉRIES FINANCEIRAS: UMA APLICAÇÃO PARA O IBOVESPA
}

\author{
Milton Barossi-Filho* \\ Jorge Alberto Achcar ${ }^{\dagger}$ \\ ROBErto MOlina de Souza $\ddagger$
}

\begin{abstract}
Resumo
Neste artigo apresentamos uma análise Bayesiana para o modelo de volatilidade estocástica (SV) e uma forma generalizada deste, cujo objetivo é estimar a volatilidade de séries temporais financeiras. Considerando alguns casos especiais dos modelos SV usamos algoritmos de Monte Carlo em Cadeias de Markov e o software WinBugs para obter sumários a posteriori para as diferentes formas de modelos SV. Introduzimos algumas técnicas Bayesianas de discriminação para a escolha do melhor modelo a ser usado para estimar as volatilidades e fazer previsões de séries financeiras. Um exemplo empírico de aplicação da metodologia é introduzido com a série financeira do IBOVESPA.
\end{abstract}

Palavras-chave: Modelo de volatilidade estocástica, Análise Bayesiana, Métodos MCMC, Séries temporais financeiras, IBOVESPA.

\begin{abstract}
In this paper, we present a Bayesian analysis for stochastic volatility models (SV) and a generalized form of this model, with the aim to estimate the volatilities of financial time series. Considering same special cases of the SV models, we use Markov Chain Monte Carlo methods and the software WinBugs to get the posterior summaries of interest for the different forms of SV models. We also introduce some Bayesian discrimination methods to choose the best model to be used to estimate the volatilities and to get predictions of the financial time series. An example of application is introduced with the Brazilian financial series IBOVESPA.
\end{abstract}

Keywords: Stochastic volatility models, Bayesian analysis, MCMC methods, financial time series, IBOVESPA.

\footnotetext{
${ }^{*}$ Faculdade de Economia, Administração e Contabilidade de Ribeirão Preto / USP, email:mbarossi@usp.br.

† Faculdade de Medicina de Ribeirão Preto / USP, email:rmolina.souza@gmail.com.

‡ Faculdade de Medicina de Ribeirão Preto / USP.
} 


\section{Introdução}

Os modelos ARCH (autoregressive conditional heteroscedasticity) introduzidos por Engle (1982) e a generalização GARCH (generalized autoregressive conditional heteroscedasticity) proposta por Bollerslev (1986) são amplamente aplicados para modelar a volatilidade de séries financeiras (Taylor 1982, Tauchen \& Pitts 1983, Bollerslev 1990, Bollerslev et al. 1992, Engle 2001). uso de modelos de volatilidade estocástica (Stochastic volatility ou SV) tem sido uma alternativa satisfatória para analisar séries temporais financeiras em comparação com os modelos usuais tipo GARCH (Kim \& Shephard 1998).

Os modelos $S V$ são mais flexíveis para modelar séries financeiras, pois assumem dois processos para os ruídos: um para as observações e outro para as volatilidades latentes. Estudos comparativos entre as classes de modelos $S V$ e do tipo $A R C H$ são amplamente discutidos na literatura (ver, por exemplo, Taylor (1994), Ghysels (1996), Shephard (1996)).

Métodos Bayesianos usando técnicas de Monte Carlo em Cadeia de Markov $(M C M C)$ são considerados para análise de séries temporais financeiras assumindo modelos $S V$ (Meyer \& Yu 2000) dada a grande dificuldade do uso das técnicas estatísticas clássicas usuais devido à complexidade da função de verossimilhança.

Neste artigo introduzimos uma análise Bayesiana para o modelo $S V$ e modificações neste modelo para estimar a volatilidade de séries financeiras no caso univariado. Também introduzimos técnicas para a discriminação de diferentes modelos usados para estimar a volatilidade de séries financeiras.

O artigo é organizado na seguinte forma: na seção 2 introduzimos modelos de volatilidade usuais e também uma generalização do modelo $S V$; na seção 3 introduzimos uma análise Bayesiana para modelos $S V$; na seção 4 introduzimos ilustrações numéricas com dados brasileiros usando o IBOVESPA e, finalmente, na seção 5 apresentamos uma discussão dos resultados obtidos.

\section{Modelos de volatilidade estocástica}

Considerando uma série financeira $P_{t}$ define-se a série transformada ou logretorno por $y_{t}=\log \left(P_{t} / P_{t-1}\right) \approx\left(P_{t}-P_{t-1}\right) / P_{t}$. Na presença de heterocedasticidade vamos assumir que o modelo para a série temporal $y_{t}$ seja dado por,

$$
y_{t}=\sigma_{t} \varepsilon_{t}
$$

em que $\varepsilon_{t}$ é um ruído suposto independente e identicamente distribuído com distribuição normal $N\left(0, \sigma_{\varepsilon}^{2}\right)$. Denotar por $H^{t}=\left(y_{t-1}, y_{t-2}, \ldots\right)$ os valores passados de $y_{t}$. A volatilidade da série $y_{t}$ é a variância condicional $\sigma_{t}^{2}=E\left(y_{t}^{2} \mid H^{t}\right)$. Também assumir que o desvio-padrão é dado pelo modelo:

$$
\sigma_{t}=\exp \left(\frac{h_{t}}{2}\right)
$$

sendo $h_{t}$ uma variável latente definida por um modelo auto regressivo $\operatorname{AR}(1)$ dado por,

$$
h_{t}=\mu+\phi\left(h_{t-1}-\mu\right)+\eta_{t}
$$

para $t=2, \ldots, N$. Vamos assumir que $h_{1}$ seja uma variável aleatória com uma distribuição conhecida $P_{1}\left(h_{1}\right)$ e $\eta_{t}$ é um ruído associado às variáveis latentes 
com uma distribuição normal $N\left(0, \sigma_{\eta}^{2}\right)$. Se $|\phi|<1$, a média e a variância nãocondicional de $h_{t}$ são respectivamente $E\left(h_{t}\right)=\mu$ e $V\left(h_{t}\right)=\sigma_{\eta}^{2} /\left(1+\phi \rho_{1}\right)$, sendo $\rho_{1}$ o coeficiente de autocorrelação entre $h_{t}$ e $h_{t-1}$.

Com as suposições (1), (2), e (3) temos:

$$
\begin{aligned}
y_{t} & \sim N\left(0, \sigma_{\varepsilon}^{2} \exp \left(h_{t}\right)\right) \\
h_{1} & \sim N\left(\mu, \sigma_{\eta}^{2}\right) \\
h_{t} \mid h_{t-1} & \sim N\left(\mu+\phi\left(h_{t-1}-\mu\right), \sigma_{\eta}^{2}\right)
\end{aligned}
$$

para $t=2,3, \ldots, N$.

Uma possível generalização para o modelo $S V$ definido pelas expressões (1) e (2) pode ser obtida definindo-se um modelo para as variáveis latentes dado por:

$$
h_{t}=\mu+\sum_{j=1}^{p} \phi_{j}\left(h_{t-j}-\mu\right)+\eta_{t}
$$

para $t=p+1, \ldots, N$, com as raízes do polinômio $\phi(B)=1-\sum_{j=1}^{p} \phi_{j} B_{j}$ fora do círculo de raio unitário ( $B$ é o operador retardo definido por $B^{k} h_{t}=h_{t-k}$ ). Denota-se o modelo definido por (1), (2) e (5) como modelo generalizado de volatilidade estocástica $(G S V)$. Neste caso temos:

$$
h_{t} \mid h_{t-1}, \ldots, h_{t-p} \sim N\left(\mu+\sum_{j=1}^{p} \phi_{j}\left(h_{t-j}-\mu\right), \sigma_{\eta}^{2}\right),
$$

para $t=2,3, \ldots, N$.

A função de verossimilhança do modelo GSV considerando as equações (1) e (1) é dada por:

$$
L=\prod_{i=1}^{T} p\left(y_{t} \mid h_{t}\right)
$$

Da equação (4) temos que:

$$
L=\prod_{i=1}^{T} \frac{1}{\sqrt{2 \pi \sigma_{\varepsilon}^{2} \exp \left(h_{t}\right)}} \exp \left(-\frac{y_{t}^{2}}{2 \sigma_{\varepsilon}^{2} \exp \left(h_{t}\right)}\right)
$$

\section{Análise Bayesiana do modelo GSV}

Para uma análise Bayesiana do modelo de volatilidade estocástica definido em (1) e (2) com as variáveis latentes definidas por (5) vamos assumir as seguintes distribuições a priori para os parâmetros $\mu=\left(\phi_{1}, \ldots, \phi_{p}\right)^{T}, \sigma_{\varepsilon}^{2}$ e $\sigma_{\eta}^{2}$ :

$$
\begin{aligned}
\phi_{j} & \sim \operatorname{Beta}\left(a_{j}, b_{j}\right), j=1, \ldots, p \\
\sigma_{\varepsilon}^{2} & \sim \operatorname{IG}\left(c_{1}, d_{1}\right) \\
\sigma_{\eta}^{2} & \sim \operatorname{IG}\left(c_{2}, d_{2}\right) \\
\mu & \sim N\left(0, e^{2}\right)
\end{aligned}
$$


sendo que $\operatorname{Beta}\left(a_{j}, b_{j}\right)$ denota uma distribuição beta com média $a_{j} /\left(a_{j}+b_{j}\right)$ e variância $a_{j} b_{j} /\left(a_{j}+b_{j}\right)^{2}\left(a_{j}+b_{j}+1\right)$; IG $(c, d)$ denota uma distribuição gama inversa com média $d /(c-1)$ e variância $d^{2} /(c-1)^{2}(c-2), c>2$ e $N\left(\mu, \sigma^{2}\right)$ denota uma distribuição normal com média $\mu$ e variância $\sigma^{2}$. Vamos considerar os hiperparâmetros $\left(a_{j}, b_{j}\right), j=1, \ldots, p,\left(c_{i}, d_{i}\right), i=1,2$ e $e^{2}$ conhecidos. Também vamos assumir independência a priori entre os parâmetros.

Fazendo $h_{l}=0$ para $l=0,-1, \ldots,-p+1$ na equação (6), a densidade condicional de $h_{t}$ dado $h_{t-1}^{(p)}=\left(h_{t-1}, \ldots, h_{t-p}\right)$, para $t=1, \ldots, N$, é dada por:

$$
p\left(h_{t} \mid h_{t-1}^{(p)}\right)=\frac{1}{\sqrt{2 \pi \sigma_{\eta}^{2}}} \exp \left\{-\frac{1}{2 \sigma_{\eta}^{2}}\left[h_{t}-\mu-\sum_{j=1}^{p} \phi_{j}\left(h_{t-j}-\mu\right)\right]^{2}\right\} .
$$

Denotando por $\Phi=\left(\phi_{1}, \ldots, \phi_{p}\right)^{T}, \theta=\left(\mu, \Phi, \sigma_{\varepsilon}^{2}, \sigma_{\eta}^{2}\right), h=\left(h_{1}, \ldots, h_{n}\right)^{T}$ e assumindo independência a priori entre os parâmetros $\mu, \Phi, \sigma_{\varepsilon}^{2}$ e $\sigma_{\eta}^{2}$, a densidade a posteriori conjunta para $\varphi=(\theta, h)$ é dada por:

$$
\begin{aligned}
\pi(\varphi \mid \mathbf{y})=\prod_{t=1}^{N} \frac{1}{\sqrt{2 \pi \sigma_{\varepsilon}^{2} \exp \left(h_{t}\right)}} \exp \left(-\frac{y_{t}^{2}}{2 \sigma_{\varepsilon}^{2} \exp \left(h_{t}\right)}\right) \\
\times \frac{1}{\sqrt{2 \pi \sigma_{\eta}^{2}}} \exp \left\{-\frac{1}{2 \sigma_{\eta}^{2}}\left[h_{t}-\mu-\sum_{j=1}^{p} \phi_{j}\left(h_{t-j}-\mu\right)\right]^{2}\right\} \pi(\theta)
\end{aligned}
$$

sendo $\mathbf{y}=\left(y_{1}, \ldots, y_{N}\right)^{T}$ e $\pi(\theta)$ é dada por:

$$
\begin{aligned}
\pi(\theta) \propto \prod_{j=1}^{p} \phi_{j}^{a_{j}-1}\left(1-\phi_{j}\right)^{b_{j}-1}\left(\sigma_{\eta}^{2}\right)^{-\frac{\left(c_{1}+1\right)}{2}} & \exp \left(-\frac{d_{1}}{\sigma_{\eta}^{2}}\right) \\
& \times\left(\sigma_{\varepsilon}^{2}\right)^{-\frac{\left(c_{2}+1\right)}{2}} \exp \left(-\frac{d_{2}}{\sigma_{\varepsilon}^{2}}\right) \exp \left(-\frac{\mu^{2}}{2 e^{2}}\right)
\end{aligned}
$$

Podemos escrever a densidade a posteriori como:

$$
\begin{aligned}
\pi(\varphi \mid \mathbf{y}) \propto\left(\sigma_{\varepsilon}^{2}\right)^{-\frac{N}{2}} & \exp \left[-\frac{1}{2} \sum_{t=1}^{N} h_{t}-\frac{1}{2 \sigma_{\varepsilon}^{2}} \sum_{t=1}^{N} y_{t}^{2} \exp \left(-h_{t}\right)\right] \\
& \times\left(\sigma_{\eta}^{2}\right)^{-\frac{N}{2}} \exp \left\{-\frac{1}{2 \sigma_{\eta}^{2}} \sum_{t=1}^{N}\left[h_{t}-\mu-\sum_{j=1}^{p} \phi_{j}\left(h_{t-j}-\mu\right)\right]^{2}\right\} \pi(\theta)
\end{aligned}
$$

Considerando a notação vetorial $\mathbf{\Phi}=\left(\phi_{1}, \ldots, \phi_{p}\right)^{T}, \mathbf{Y}=\left(y_{1}^{2}, \ldots, y_{N}^{2}\right)^{T}, \mathbf{h}=\left(h_{1}, \ldots, h_{N}\right)^{T}$, $\mathbf{E}(\mathbf{h})=\left[\exp \left(-h_{1}\right), \ldots, \exp \left(-h_{N}\right)\right]^{T}$ e a matriz $\mathbf{X}$ dada por:

$$
\mathbf{X}=\left(\begin{array}{cccc}
h_{0} & h_{-1} & \cdots & h_{-p+1} \\
h_{1} & h_{0} & \cdots & h_{-p+2} \\
\vdots & \vdots & \ddots & \vdots \\
h_{p} & h_{p-1} & \cdots & h_{1} \\
h_{p+1} & h_{p} & \cdots & h_{2} \\
\vdots & \vdots & \ddots & \vdots \\
h_{N-1} & h_{N-2} & \cdots & h_{N-p}
\end{array}\right)_{N \times p}
$$


podemos escrever a densidade a posteriori na forma,

$$
\begin{aligned}
\pi(\varphi \mid \mathbf{y}) \propto\left(\sigma_{\varepsilon}^{2}\right)^{-\frac{N}{2}} \exp & {\left[-\frac{1}{2} \mathbf{1}^{T} \mathbf{h}-\frac{1}{2 \sigma_{\varepsilon}^{2}} \mathbf{Y}^{T} \mathbf{E}(\mathbf{h})\right] } \\
\times & \left(\sigma_{\eta}^{2}\right)^{-\frac{N}{2}} \exp \left[-\frac{1}{2 \sigma_{\eta}^{2}}(\mathbf{h}-\mu-\mathbf{X} \mathbf{\Phi})^{T}(\mathbf{h}-\mu-\mathbf{X \Phi})\right] \pi(\theta)
\end{aligned}
$$

em que $1=(1, \ldots 1)^{T}$ é o vetor $N \times 1$ de $1^{\prime} s$ e $\mu$ é um vetor $N \times 1$ de $\mu^{\prime} s$.

Para gerar amostras da distribuição a posteriori conjunta para $\varphi=(\theta, h)$ usamos métodos MCMC com o algoritmo Gibbs Sampling (ver, por exemplo, Gelfand \& Smith (1990)) ou o algoritmo Metropolis-Hastings (ver, por exemplo, Smith \& Roberts (1993)). Essas amostras são geradas a partir das distribuições condicionais $\pi\left[\theta_{j} \mid \theta_{(j)}, \mathbf{y}\right]$, em que $\theta_{(j)}$ denota o vetor de todas as componentes de $\theta$ exceto o $j$-ésimo componente.

Uma grande simplificação é obtida usando o software WinBugs (Spiegelhalter et al. 1999), no qual apenas necessitamos definir a distribuição para os dados e as distribuições a priori para os parâmetros do modelo.

Resultados similares são obtidos considerando casos especiais do modelo $G S V \operatorname{com} p=1, \phi_{j}=0$ para $j=2, \ldots, p(S V$ usual $)$ e $\sigma_{\varepsilon}^{2}=1$ ou $\sigma_{\eta}^{2}=1$.

\section{Critérios de seleção de modelos}

No contexto bayesiano, há vários critérios para escolha do modelo de volatilidade estocástica que melhor se ajusta a uma série financeira (ver, por exemplo, Berg et al. (2004)). Dentre estes, vamos usar aqui os seguintes critérios:

\subsection{Critério de Informação Bayesiana (BIC)}

O BIC (Bayesian Information Criterion) é um critério de seleção de modelo proposto por Schwarz (1978) e modificado por Carlin \& Louis (2000) para ser aplicado considerando a densidade a posteriori dos parâmetros do modelo ajustado. Este critério pondera entre a função log-verossimilhança maximizada e o número de parâmetros do modelo. O melhor modelo é aquele que apresenta o maior valor do BIC dado por,

$$
B I C=E[\ln L(\theta)]-\frac{1}{2} p \ln (N)
$$

em que $E[\ln L(\theta)]$ é o valor esperado tomado com relação a densidade a posteriori, da função log-verossimilhança, $p$ é a dimensão do vetor de parâmetros e $N$ é o tamanho amostral.

\subsection{Critério Desvio-Informação (DIC)}

O DIC (Deviance Information Criterion) é uma generalização do BIC. Este critério é particularmente usual nos problemas Bayesianos de seleção de modelos para os quais amostras da distribuição a posteriori dos parâmetros dos modelos foram obtidas por simulação de Monte Carlo em Cadeias de Markov $(M C M C)$. Semelhante ao BIC este critério é uma aproximação assintótica para amostras grandes e é valido quando a distribuição a posteriori é aproximadamente uma distribuição normal multivariada. 
Define-se o desvio como:

$$
D(\theta)=-2 \ln L(\theta)+C
$$

em que $\theta$ é o vetor de parâmetros desconhecidos do modelo; $L(\theta)$ a função de verossimilhança e $C$ é uma constante que se cancela e, portanto não precisa ser conhecida na comparação de dois modelos. O critério DIC introduzido por Spiegelhalter et al. (2002) é dado por:

$$
D I C=D(\hat{\theta})+2 p_{D}
$$

sendo $D(\hat{\theta})$ o desvio avaliado na média a posteriori e $p_{D}$ é o número efetivo de parâmetros no modelo, que é dado por $p_{D}=\bar{D}-D(\hat{\theta}$, em que $\bar{D}=E[D(\theta)]$ é o desvio médio a posteriori que mede a qualidade do ajuste do modelo aos dados. Valores menores para DIC indicam melhores modelos, podendo estes serem negativos. O DIC é usado para a discriminação de diferentes modelos propostos para a análise de séries financeiras.

\subsection{Fator de Bayes $\left(B_{i j}\right)$}

Este critério compara dois modelos $M_{1}$ e $M_{2}$ usando o conceito de densidade marginal ou verossimilhança marginal. O modelo $M_{1}$ é melhor do que o modelo $M_{2}$ se $P\left(D \mid M_{2}\right)<P\left(D \mid M_{1}\right)$ em que $P\left(D \mid M_{l}\right), l=1,2$ é a verossimilhança marginal e, esta é dada por:

$$
P\left(D \mid M_{l}\right)=\int_{\theta_{l}} L\left(D \mid \theta_{l}, M_{l}\right) \pi_{0}\left(\theta_{l} \mid M_{l}\right) d \theta_{l}
$$

sendo $L\left(D \mid \theta_{l}, M_{l}\right), l=1,2$ a função de verossimilhança dos dados sob a hipótese do modelo $M_{l}$ e $\pi_{0}\left(\theta_{l} \mid M_{l}\right)$ é a densidade a priori para os parâmetros $\theta_{l}$. O fator de Bayes é dado pela razão,

$$
B_{i j}=\frac{P\left(D \mid M_{i}\right)}{P\left(D \mid M_{j}\right)}
$$

Uma modificação do fator de Bayes proposta por Aitkin (1991) permite que a integral (7) seja obtida pelo estimador de Monte Carlo com as amostras geradas via $M C M C$ da densidade a posteriori $\pi_{0}\left(\theta_{l} \mid M_{l}\right)$. A verossimilhança marginal pode ser estimada por,

$$
\overline{P\left(D \mid M_{l}\right)}=\frac{1}{M} \sum_{j=1}^{M} L\left(\theta_{l}^{(j)} \mid M_{l}\right),
$$

para $l=1$,2; em que $M$ é o número de amostras geradas de $\pi_{0}\left(\theta_{l} \mid M_{l}\right)$.

Outro critério de discriminação proposto por Raftery (1995), (ver também Raftery et al. (2006)) é dado pela média harmônica da verossimilhança,

$$
\overline{f\left(D \mid M_{l}\right)}=\left[\frac{1}{M} \sum_{j=1}^{M} \frac{1}{L\left(D \mid \theta_{l}^{(j)}, M_{l}\right)}\right]^{-1}
$$

para $l=1,2$. Os critérios de seleção apresentados nesta seção são usados para selecionar o melhor modelo que se ajusta ao log-retorno da série financeira considerada na próxima seção. 


\section{Análise Bayesiana do IBOVESPA}

Em linhas gerais, o IBOVESPA é o indicador de desempenho médio mais importante do mercado acionário brasileiro, constituindo uma carteira de mercado amplamente utilizada. O IBOVESPA refere-se ao índice de desempenho médio de um conjunto das ações mais negociadas em determinado período de tempo, na Bolsa de Valores de São Paulo. A importância desse índice em mercado financeiro reflete-se, basicamente, em três de suas naturezas: tradição em representatividade das negociações do mercado acionário brasileiro, abrangência da série temporal, que é ininterrupta desde fins dos anos 60 e, pela sua ampla utilização e aceitação no mercado financeiro como benchmark.

De acordo com BOVESPA (2007), o IBOVESPA é o valor atual em moeda corrente de uma carteira teórica constituída em 02 de janeiro de 1968, a partir da aplicação de um valor inicial hipotético admitindo-se nulas quaisquer aplicações adicionais de recursos na carteira. O IBOVESPA não reflete apenas o retorno de uma carteira teórica de mercado, mas o impacto da distribuição de proventos, por isso avalia, de fato, o retorno total das ações das empresas que constituem a carteira.

Ainda, de acordo com BOVESPA (2007), o índice é composto, em termos metodológicos, por ações de empresas negociadas na Bolsa de Valores de São Paulo que tenham atendido, cumulativamente, a três critérios fundamentais: pertencer ao conjunto de ações que representem, somados, $80 \%$ em termos de negociabilidade; participação, em termos de volume de negociação, superior a $0,1 \%$ do total e ter sido negociada em mais de $80 \%$ dos pregões do período. O período referencial ou base constitui-se dos doze meses anteriores à formação da carteira. Claramente, a negociabilidade é representativa da participação do título nos pregões à vista ajustada pelo tamanho da amostra admitido. Finalmente, a exclusão de uma ação da composição da carteira de mercado justifica-se, desde que a mesma não seja capaz de atender a dois dos critérios acima, pelo menos.

Portanto, por definição o IBOVESPA é a soma total dos valores ponderados teóricos das ações que o compõem. Assim, em qualquer momento de tempo, o índice é definido pela quantidade teórica da ação ponderada pelo último preço de referência, ou negociado em mercado da mesma e pode ser escrito como

$$
I_{t}=\sum_{i=1}^{n} P_{i t} Q_{i t}
$$

em que: $n$ é o número total de ações que constitui o índice; $P_{i t}$ é último preço da ação $i$ no instante de tempo $t$ e $Q_{i t}$ é a quantidade teórica da ação $i$ na carteira no instante de tempo $t$.

Em termos amostrais, o logaritmo do retorno do IBOVESPA foi calculado em bases amostrais semanais e centralizado na média. O início da amostra toma por referencial a primeira data de pregão da semana em que o Plano Real foi implantado, 04 de julho de 1994, seguindo pelos próximos treze anos, até o dia 18 de fevereiro de 2008, totalizando 712 semanas de negociação do índice em bolsa.

Considerar os log-retornos $y_{t}=\ln \left(I_{t+1} / I_{t}\right), t=1, \ldots, 712$ em que $I_{t}$ são índices médios semanais e centralizados em torno de sua média. O gráfico da série $y_{t}$ é dado na Figura 1. 


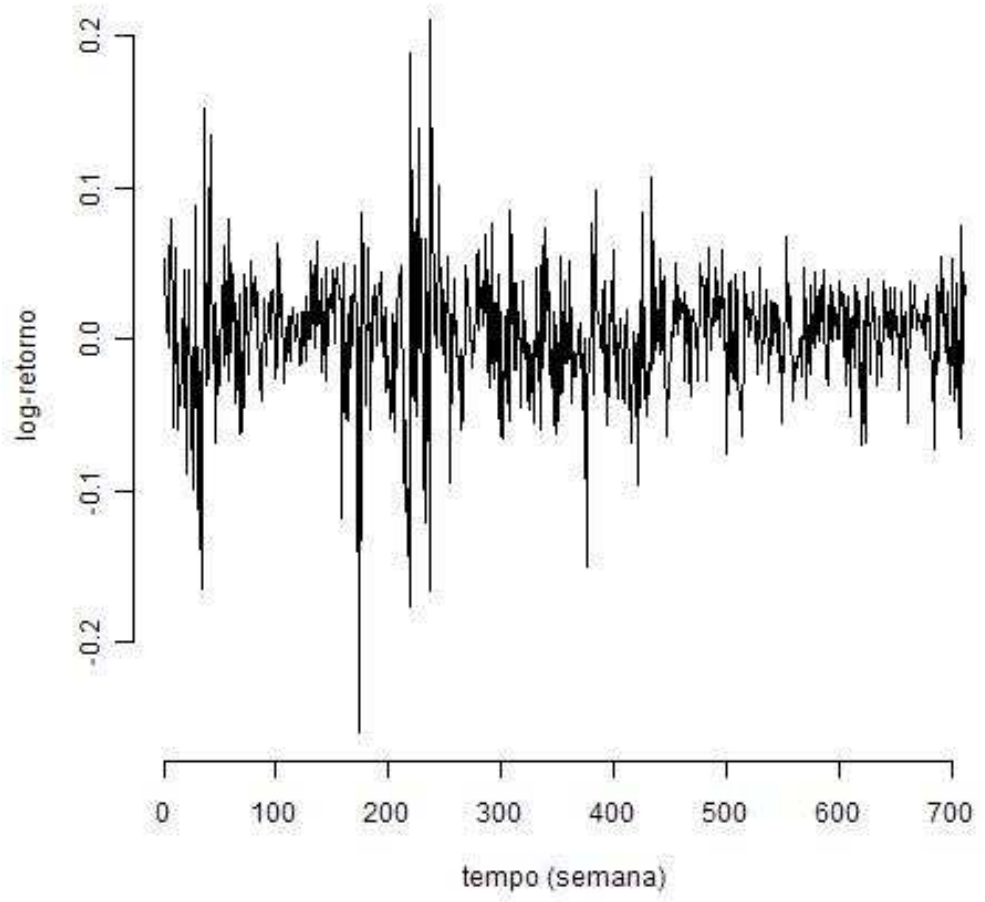

Figura 1: Série log-retornos do IBOVESPA centralizada na média

Claramente, o comportamento do retorno do IBOVESPA ao longo do período é marcado por três momentos distintos. Primeiro, um movimento de volatilidade expressiva até a primeira metade do período que compreende as 100 primeiras semanas da amostra, que se segue à implantação do Plano Real. Tratam-se, na verdade, de procedimentos usuais em momentos como esses no sentido de extrair as informações relevantes de política econômica e seus impactos sobre o apreçamento das ações das principais empresas refletido no índice de mercado.

Segundo, após um breve período de calmaria em termos de volatilidade do retorno do índice segue-se, novamente, ao final do intervalo entre a centésima e ducentésima semanas um período crítico de excesso de volatilidade que se estende ao próximo intervalo. Esse movimento acalma-se apenas depois da ducentésima qüinquagésima semana de pregão, cujas conseqüências se fazem refletir sobre o regime cambial brasileiro adotado a partir do início de 1999. As crises asiática e russa explicam o perfil de excessiva volatilidade que se delineia nesse período. As conseqüências econômicas e financeiras dessas duas crises gêmeas foram sentidas nos principais indicadores econômicos de desempenho da economia brasileira no período levando, claramente, as autoridades monetárias a optarem por um regime de taxa de câmbio flutuante em janeiro de 1999.

Finalmente, em terceiro lugar, da trecentésima semana até o final do pe- 
ríodo amostral, a volatilidade refletida pelo índice é menor e decrescente. A despeito dessa característica marcante dos mercados financeiros brasileiros que se inicia no ano de 2002 é possível perceber um momento de stress que antecede as eleições de 2002. O desconhecimento do mercado financeiro em relação ao provável comportamento futuro da política econômica de um governo liderado pelo PT levou, inicialmente, os agentes econômicos a especularem sobre prazos e possíveis tentativas de descumprimentos de compromissos assumidos no governo que se findava. No entanto, nada reproduziria o perfil de volatilidade excessiva da década anterior, marcada por desajustes externos e a implementação de políticas econômicas ortodoxas que definiram um perfil conservador, além de impor custos eleitorais significativos ao governo que terminava o mandato.

Como a amostra não inclui a totalidade do episódio da crise do mercado de hipotecas sub-prime norte-americano, o comportamento do IBOVESPA próximo à $712 \underline{a}$ semana reflete pouca volatilidade, comparado aos períodos imediatamente anteriores.

Para analisar os dados consideramos inicialmente o modelo de volatilidade estocástica ( $S V)$ definido pelas equações de (1), a (4), assumindo $\sigma_{\varepsilon}^{2}=1$ e as distribuições a priori $\phi \sim \operatorname{Beta}(1,1) ; \tau_{\eta}=1 / \sigma_{\eta}^{2} \sim \operatorname{gama}(1,1)$ e $\mu \sim N(0,100)$, em que gama $(a, b)$ denota uma distribuição gama com média $a / b$ e variância $a / b^{2}$. A escolha de uma distribuição gama $(a, b)$ para $\tau_{\eta}$ corresponde à escolha de uma distribuição gama-inversa para $\sigma_{\eta}^{2}$. Vamos denotar este modelo como modelo 1A. Observe que a distribuição a priori escolhida para $\mu$ apresenta uma variância grande, o que leva a uma priori não-informativa.

Os sumários a posteriori para os parâmetros do modelo apresentados na Tabela 1 foram obtidos a partir da geração de 1000 amostras, simuladas com o algoritmo Gibbs Sampling, da distribuição a posteriori conjunta escolhidas de 100 em 100 para a obtenção de amostras não correlacionadas e após descartar as 5000 primeiras amostras geradas (burn-in samples) com intuito de eliminar o efeito dos valores iniciais para os parâmetros. Para simulação dessas amostras utilizamos o software WinBugs (Spiegelhalter et al. 1999).

Sumários a posteriori para os parâmetros do modelo $S V$ assumindo $\sigma_{\varepsilon}^{2}$ desconhecido (modelo 1B) também são apresentados na Tabela 1. Esses sumários a posteriori foram obtidos de forma similar aos obtidos para o modelo $1 \mathrm{~A}$, considerando as mesmas distribuições a priori para $\phi$, $\mu$ e $\tau_{\eta}=1 / \sigma_{\eta}^{2}$; para $\tau_{\varepsilon}=1 / \sigma_{\varepsilon}^{2}$ consideramos uma distribuição a priori gama $(100,100)$.

De forma similar apresentamos na Tabela 1, os sumários a posteriori para os parâmetros do modelo de volatilidade estocástica generalizado (GSV) definido por (1), (2) e (5) com $p=2, \sigma_{\varepsilon}^{2}=1$ (modelo $2 \mathrm{~A}$ ) e assumindo $\sigma_{\varepsilon}^{2}$ desconhecido (modelo $2 \mathrm{~B}$ ).

Para o modelo $2 \mathrm{~A}$ assumimos as distribuições a priori $\phi_{1} \sim \operatorname{Beta}(1,1), \phi_{2} \sim$ $\operatorname{Beta}(1,1), \tau_{\eta}=1 / \sigma_{\eta}^{2} \sim \operatorname{gama}(1,1)$ e $\mu \sim N(0,100)$.

Para o modelo $2 \mathrm{~B}$ assumimos uma distribuição a priori para $\tau_{\varepsilon}=1 / \sigma_{\varepsilon}^{2} \sim$ $\operatorname{gama}(1,1)$ e as distribuições a priori $\phi_{1} \sim \operatorname{Beta}(1,1), \phi_{2} \sim \operatorname{Beta}(1,1), \tau_{\eta}=$ $1 / \sigma_{\eta}^{2} \sim \operatorname{gama}(100,100)$ e $\mu \sim N(0,100)$.

Com a finalidade de comparação com modelos usuais $A R C H$ assumimos o modelo $1 \mathrm{~A} \operatorname{com} \varepsilon_{t}$ independentes e identicamente distribuídos com distribui- 
ção normal $N(0,1)$ e $\sigma_{t}^{2}$ modelado como um $\operatorname{ARCH}(2)$, dado por:

$$
\begin{aligned}
y_{t} & =\sigma_{t} \varepsilon_{t} \\
\sigma_{t}^{2} & =\alpha_{0}+\alpha_{1} y_{t-1}^{2}+\alpha_{2} y_{t-2}^{2}
\end{aligned}
$$

$\operatorname{com} \varepsilon_{t} \stackrel{\text { i.i.d. }}{\sim} N(0,1), \alpha_{0}>0, \alpha_{j} \geq 0, j=1,2$ e devemos assegurar que $\left(\alpha_{1}+\alpha_{2}\right)<$ 1. Para este modelo foram adotadas distribuições a priori $\alpha_{j} \sim \operatorname{gama}(1 ; 1)$, $j=0,1,2$. Sumários a posteriori para $\alpha_{j}, j=0,1,2$ foram obtidos a partir de simulação de 1000 amostras geradas, com o algoritmo Gibbs Sampling, tomadas de 100 em 100 após um burn-in de tamanho 5000, da distribuição a posteriori para $\alpha_{j}, j=0,1,2$. Estes sumários são apresentados na tabela 1.

Outro modelo usual que podemos comparar com os modelos apresentados neste texto é o modelo GARCH. Para tanto, novamente assumimos o modelo $1 \mathrm{~A}$ com $\varepsilon_{t}$ independentes e identicamente distribuídos com distribuição normal $N(0,1)$ e $\sigma_{t}^{2}$ modelado como um $\operatorname{GARCH}(1,1)$, dado por:

$$
\begin{aligned}
y_{t} & =\sigma_{t} \varepsilon_{t} \\
\sigma_{t}^{2} & =\alpha_{0}+\alpha_{1} y_{t-1}^{2}+\beta_{1} \sigma_{t-1}^{2}
\end{aligned}
$$

$\operatorname{com} \varepsilon_{t} \stackrel{\text { i.i.d. }}{\sim} N(0,1), \alpha_{0}>0, \alpha_{1}$ e $\beta_{1} \geq 0$ e devemos assegurar que $\left(\alpha_{1}+\beta_{1}\right)<1$. Para este modelo foram adotadas distribuições a priori $\alpha_{j} \sim \operatorname{gama}(1 ; 1), j=0,1$ e $\beta_{1} \sim \operatorname{gama}(1 ; 1)$. Sumários a posteriori para $\alpha_{j}, j=0,1$ e $\beta_{1}$ foram obtidos da mesma forma que para o modelo $A R C H$ e também são apresentados na tabela 1 .

Na Tabela 2 apresentamos as estimativas de Monte Carlo a posteriori dos critérios de seleção introduzidos na seção 4 para os 6 modelos ajustados para os dados.

Observando as estimativas de Monte Carlo para o DIC considerando o modelo $1 \mathrm{~A}(D I C=-2708,88)$ e o modelo $2 \mathrm{~A}(D I C=-2712,08)$, e o modelo $1 \mathrm{~B}$ $(D I C=-2708,17)$ e o modelo $2 \mathrm{~B}(D I C=-2712,91)$, concluímos que os modelos $2 \mathrm{~A}$ e $2 \mathrm{~B}$ (em que são considerados $\phi_{1}$ e $\phi_{2}$ ) são melhores ajustados que os modelos $1 \mathrm{~A}$ e $1 \mathrm{~B}$.

As estimativas de Monte Carlo obtidas para o DIC considerando os modelos $\mathrm{ARCH}(2)$ e $\mathrm{GARCH}(1,1)$ são dados por $D I C=-2588,27$ e $D I C=-2624,33$. Desta forma, concluímos que modelos $S V$ ou GSV levam a melhores estimativas para a volatilidade das séries financeiras, pois temos melhores ajustes para estes modelos (menores valores para DIC). Podemos chegar à mesma conclusão analisando o Fator de Bayes dado na Tabela 3.

A análise do critério BIC estimado a posteriori e apresentado na Tabela 2 leva às mesmas conclusões do critério $D I C$ quando diz respeito à comparação de modelos $S V$ ou $G S V$ com os modelos usualmente assumidos ( $A R C H$ e $G A R C H)$. Porém, entre modelos $S V$ e GSV, pelo fato do critério BIC ser mais rigoroso na penalização com relação ao número de parâmetros, os modelos $1 \mathrm{~A}$ e $1 \mathrm{~B}$ (que tem um parâmetro a menos que os modelos $2 \mathrm{~A}$ e $2 \mathrm{~B}$ ) apresentam maiores valores de $B I C$.

Cada linha da Tabela 3 mostra o fator de Bayes a posteriori de cada modelo com relação aos demais. Notamos que os modelos $A R C H$ e GARCH são superados por todos os modelos generalizados de volatilidade estocástica (GSV). 
Tabela 1: Sumários a posteriori dos modelos analisados (d. p.: desviopadrão; ICr: Intervalo de credibilidade)

\begin{tabular}{ccccc}
\hline Modelo & $\theta$ & médias & d.p. & $I C_{r}$ \\
\hline \multirow{2}{*}{$\mathrm{A}_{\varepsilon}^{2}=1$} & $\mu$ & $-6,73$ & 0,16 & $(-7,04 ;-6,39)$ \\
& $\theta$ & 0,91 & 0,03 & $(0,83 ; 0,95)$ \\
& $\sigma_{\eta}^{2}$ & 8,14 & 2,01 & $(4,80 ; 12,39)$ \\
\hline \multirow{2}{*}{$1 \mathrm{~B}$} & $\sigma_{\varepsilon}^{2}$ & 0,99 & 0,10 & $(0,81 ; 1.19)$ \\
$\sigma_{\varepsilon}^{2}$ desc. & $\mu$ & $-6,72$ & 0,19 & $(-7,07 ;-6,37)$ \\
& $\theta$ & 0,90 & 0,03 & $(0,83 ; 0,95)$ \\
& $\sigma_{\eta}^{2}$ & 7,87 & 2,00 & $(4,62 ; 12,22)$ \\
\hline \multirow{2}{*}{$2 \mathrm{~A}$} & $\mu$ & $-6,71$ & 0,20 & $(-7,08 ;-6,27)$ \\
$\sigma_{\varepsilon}^{2}=1$ & $\phi_{1}$ & 0,4 & 0,15 & $(0,15 ; 0,73)$ \\
& $\phi_{2}$ & 0,51 & 0,15 & $(0,19 ; 0,77)$ \\
& $\sigma_{\eta}^{2}$ & 6,05 & 1,67 & $(3,50 ; 10,01)$ \\
\hline \multirow{2}{*}{$2 \mathrm{~B}$} & $\sigma_{\varepsilon}^{2}$ & 0,99 & 0,10 & $(0,81 ; 1.21)$ \\
$\sigma_{\varepsilon}^{2}$ desc. & $\mu$ & $-6,69$ & 0,22 & $(-7,12 ;-6,25)$ \\
& $\phi_{1}$ & 0,4 & 0,15 & $(0,15 ; 0,74)$ \\
& $\phi_{2}$ & 0,51 & 0,16 & $(0,17 ; 0,78)$ \\
& $\sigma_{\eta}^{2}$ & 5,97 & 1,51 & $(3,37 ; 9,43)$ \\
\hline \multirow{2}{*}{$A R C H(2)$} & $\alpha_{0}$ & 0,001 & $8,42 \times 10^{-5}$ & $\left(9,61 \times 10^{-4} ; 0,001\right)$ \\
& $\alpha_{1}$ & 0,26 & 0,06 & $(0,5 ; 0,40)$ \\
& $\alpha_{2}$ & 0,08 & 0,04 & $(0,03 ; 0,16)$ \\
\hline \multirow{2}{*}{ GARCH(1,1) } & $\alpha_{0}$ & $2,19 \times 10^{-4}$ & $6,46 \times 10^{-4}$ & $\left(1,29 \times 10^{-4} ; 3,72 \times 10^{-4}\right)$ \\
& $\alpha_{1}$ & 0,17 & 0,04 & $\left(1,29 \times 10^{-4} ; 3,72 \times 10^{-4}\right)$ \\
& $\beta_{1}$ & 0,70 & 0,06 & $(0,57 ; 0,79)$ \\
\hline & & & &
\end{tabular}

Tabela 2: Critérios de seleção aplicados ao modelo

\begin{tabular}{lccc}
\hline Modelos & $E[\ln L(\theta)]$ & DIC & BIC \\
\hline 1A & 1431,86 & $-2708,88$ & 1428,58 \\
1B & 1432,68 & $-2708,17$ & 1429,39 \\
2A & 1432,53 & $-2712,08$ & 1425,97 \\
2B & 1431,96 & $-2712,91$ & 1425,39 \\
ARCH & 1296,89 & $-2588,27$ & 1295,71 \\
GARCH & 1315,15 & $-2624,33$ & 1308,58 \\
\hline
\end{tabular}

Tabela 3: Fator de Bayes a posteriori para dois modelos $M_{i}$ e $M_{j}$

\begin{tabular}{|c|c|c|c|c|c|}
\hline \multirow{2}{*}{$\frac{\text { Modelos }}{1 \mathrm{~A}(i=1)}$} & \multicolumn{3}{|c|}{$B_{i, j}=P\left(D \mid M_{i}\right) / P\left(D \mid M_{j}\right), i \neq j$} & \multirow{2}{*}{$\frac{A R C H(j=5)}{B_{15}=1,149}$} & \multirow{2}{*}{$\frac{G A R C H(j=6)}{B_{16}=1,120}$} \\
\hline & $B_{12}=1,000$ & $B_{13}=0,997$ & $B_{14}=0,999$ & & \\
\hline $1 \mathrm{~B}(i=1)$ & $B_{22}=1,000$ & $B_{23}=0,997$ & $B_{24}=0,999$ & $B_{25}=1,149$ & $B_{26}=1,120$ \\
\hline $2 \mathrm{~A}(i=1)$ & $B_{32}=1,000$ & $B_{33}=0,997$ & $B_{34}=0,999$ & $B_{35}=1,149$ & $B_{36}=1,120$ \\
\hline $2 \mathrm{~A}(i=1)$ & $B_{42}=1,000$ & $B_{43}=0,997$ & $B_{44}=0,999$ & $B_{45}=1,149$ & $B_{46}=1,120$ \\
\hline$A R C H(i=1)$ & $B_{52}=1,000$ & $B_{53}=0,997$ & $B_{5}=0,999$ & $B_{55}=1,149$ & $B_{56}=1,120$ \\
\hline
\end{tabular}


Na Figura 2 temos os gráficos das estimativas de Monte Carlo para as médias a posteriori das volatilidades estocásticas considerando os 6 modelos propostos. Uma comparação dos gráficos $(a, b, c, e d)$ com os $(e, f)$ deixa claro a diferença na qualidade das estimativas da variação da volatilidade (heterocedasticidade localizada nas primeiras 300 semanas) estimada com cada modelo.

\section{Discussão dos Resultados}

Comparando-se as volatilidades estimadas pelos modelos de volatilidade da classe $A R C H$ e GARCH e modelos de volatilidade estocástica da classe generalizada, em que procedimentos Bayesianos foram aplicados para estimação é possível delinear dois conjuntos de observações.

Primeiro, os modelos de volatilidade estocástica (SV) mostraram resultados superiores a modelos da classe $A R C H$ e GARCH. A veracidade da afirmação pode ser confirmada tanto pelos resultados estatísticos obtidos, como pelas estimativas dos critérios de informação calculados. Além disso, dentre os modelos de volatilidade estocástica, dependendo do critério utilizado, ora tem-se um melhor ajuste para os modelos de segunda ordem $\left(\phi_{1}\right.$ e $\left.\phi_{2}\right)$ (critérios DIC e Fator de Bayes) ora tem-se melhores ajustes para os modelos de primeira ordem (critério BIC). Nota-se também que, pelo fato das médias $a$ posteriori de $\sigma_{\varepsilon}^{2}$, para os modelos em que esta variável é desconhecida, estarem sempre próximas de 1 não foi possível uma melhor discriminação com relação aos modelos em que $\sigma_{\varepsilon}^{2}$ foi considerado igual a 1 .

Segundo, observando-se a figura $2(a, b, c, \mathrm{e} d)$ verificam-se as estimativas de Monte Carlo para os modelos de volatilidade estocástica generalizada. Essas estimativas estão dispostas em semanas, revelando alguns padrões de comportamento esperados pela teoria econômica (Blanchard \& Fisher 1989, Ross et al. 2005) e outros surpreendentes, que merecem uma melhor elaboração de justificativas. Teoricamente esperados são os comportamentos gerais padrão das estimativas de volatilidade refletindo, basicamente, os principais eventos macroeconômicos que marcaram a economia brasileira nos últimos treze anos. Isto é, as crises financeiras internacionais e brasileira de 1997, 1998 e 1999, respectivamente, e o evento das eleições de 2002. No entanto, as estimativas de volatilidade provenientes dos modelos de volatilidade estocástica produzem informações, que analisadas em nível desagregado, caracterizam padrões de comportamento surpreendentes, principalmente quando comparamos analiticamente esse modelo às estimativas de um modelo da classe $A R C H$. Claramente, a amplitude de variação da volatilidade do IBOVESPA é menor, além de decrescente a partir de fins de 1999. Ainda, o padrão descendente é reforçado a partir da $500^{a}$ semana de pregão; isto é a partir de 2004 e atingindo um ponto de mínimo próximo à última semana da amostra.

Porém, o padrão volátil que mais chama atenção, a partir das estimativas dos modelos de volatilidade estocástica é uma aparente repetição sazonal de excessos de volatilidade com freqüência média de 100 semanas. Isto é, com intervalo médio de 100 semanas, o índice torna-se mais volátil, mesmo diante de uma tendência geral descendente da volatilidade. É possível levantarmos algumas hipóteses à explicação de um padrão repetitivo como tal, principalmente aquelas calcadas em fatores econômicos, financeiros ou políticos. Entretanto, como o IBOVESPA é também um indicador de desempenho 


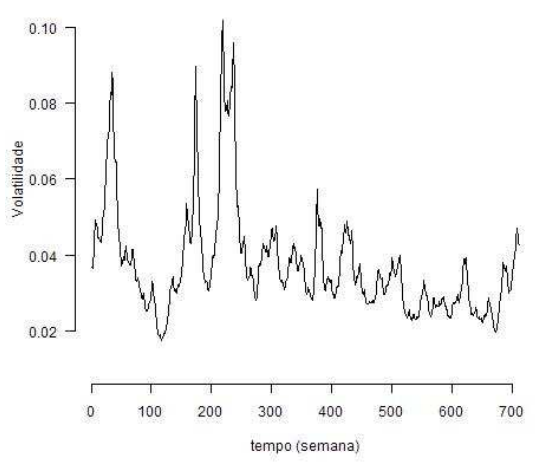

(a) Modelo $1 \mathrm{~A}$

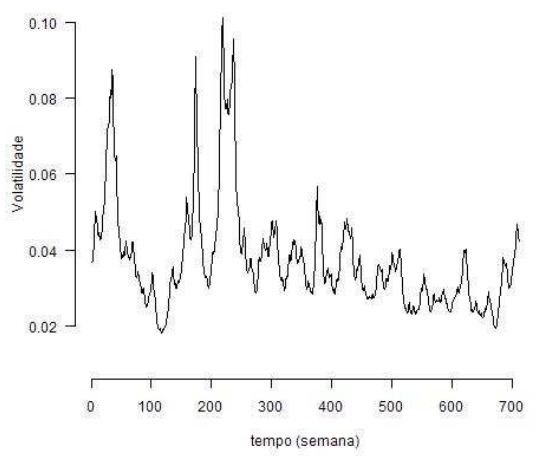

(c) Modelo 2A

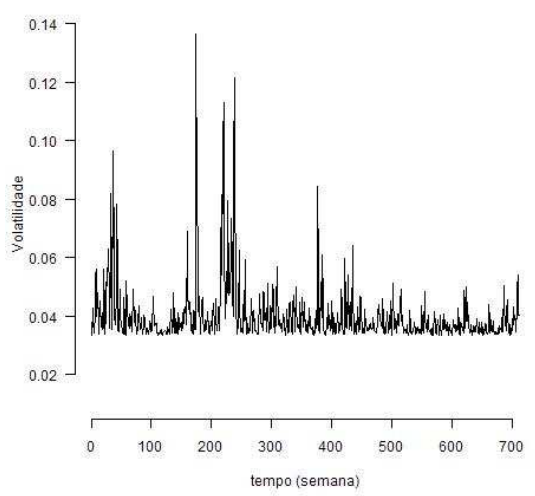

(e) Modelo $A R C H$

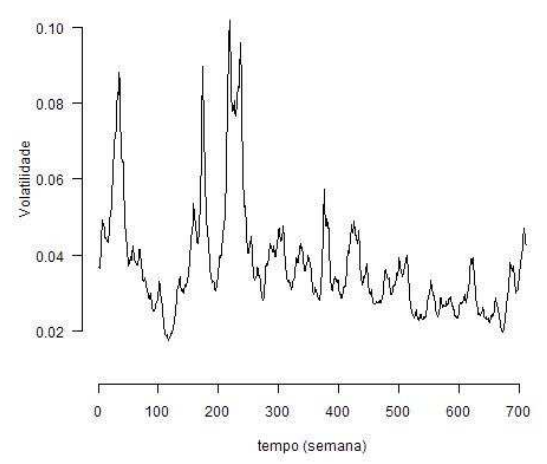

(b) Modelo 1B

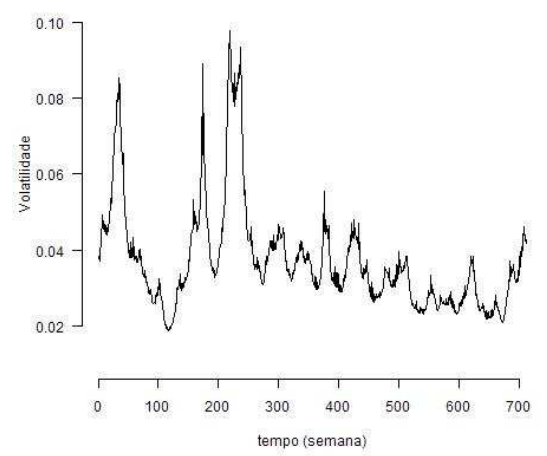

(d) Modelo 2B

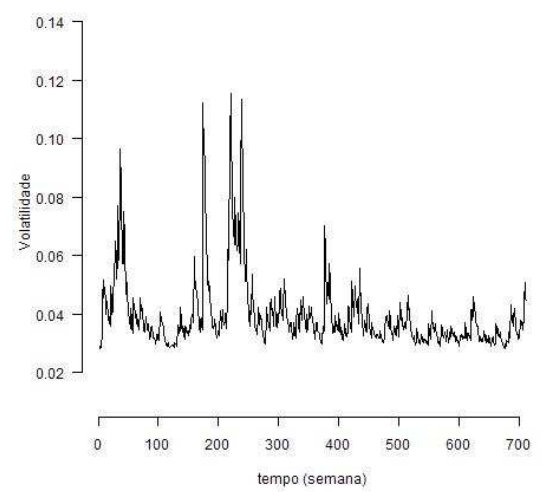

(f) Modelo GARCH

Figura 2: Volatilidade estocástica para os 6 modelos considerados. 
econômico e financeiro de um conjunto de empresas, que se justificam pela sua capacidade em gerar renda à sociedade, mais do que um mero indicador especulativo, a busca de hipóteses explicativas calcadas na teoria econômica torna-se premente. Os papéis desempenhados pelas crises econômicas e financeiras foram fundamentais, porém as influências das mesmas cessaram a partir de meados de 2003 e os padrões voláteis continuaram a se manifestar.

Abre-se ainda a possibilidade de se associar o comportamento delineado pela Figura2 $(a, b, c, e d)$, à política monetária seguida pelo governo a partir de 2003. Na verdade, tanto as elevações de taxas de juros ocorridas entre 2003 e 2004 foram relevantes, como o processo reverso, a partir de meados de 2005 e o mercado de capitais, representado por um índice agregado como o IBOVESPA reflete tanto movimentos especulativos de curto prazo, quanto perspectivas dos ciclos dos negócios e decisões de investimento de longo prazo das empresas de capital aberto. Logo, associar as evidências encontradas apenas às nuances da política monetária é, certamente, deixar de levantar hipóteses mais robustas à explicação do fenômeno.

Teoricamente podemos associar os efeitos voláteis sobre o IBOVESPA que se manifestam, em média, em períodos sincronizados com intervalo de 100 semanas aos ciclos econômicos de crescimento e desenvolvimento econômicos que levam, certamente, a novas aberturas de capital e buscas crescentes de recursos para financiamento em mercados de capital (Blanchard \& Fisher 1989, Ross et al. 2005). Sem dúvida, uma fonte de recursos das mais baratas ao financiamento do crescimento e desenvolvimento econômicos das empresas brasileiras. Neste sentido, as empresas com ações negociadas na Bolsa de Valores de São Paulo tornaram-se mais atrativas reproduzindo aspectos dos ciclos dos negócios que caracterizam todas as economias capitalistas modernas.

Finalmente, cabe ressaltar que a despeito do estágio de crescimento e desenvolvimento econômicos atingidos na última década e meia no Brasil, a política monetária norte-americana ainda é um fator de influência exógena sobre o mercado de capitais brasileiro. A crise gerada a partir do episódio de 11 de setembro de 2001 Nova York, cuja resposta foi uma queda generalizada e prolongada das taxas de juros nos mercados de fundos emprestáveis norte-americanos e europeus produziu um excesso de liquidez internacional, parecido em magnitude apenas aquele verificado em fins dos anos 60 .

Portanto, a queda prolongada das taxas de juros externas, a recuperação dos fundamentos econômicos da economia brasileira na última década e meia, aliadas às estabilidades política e da política econômica garantiram a tendência decrescente no espectro de volatilidade em termos gerais. Especificamente, o desempenho das empresas com ações negociadas em bolsa engajadas em ciclos econômicos, teoricamente caracterizados no período verificado apontam uma característica própria aos mesmos refletida, no longo prazo, pelo comportamento do IBOVESPA. 


\section{Referências Bibliográficas}

Aitkin, M. (1991), 'Posterior bayes factors', Journal of the Royal Statistical Society 53, 111-142.

Berg, A., Meyer, R. \& Yu, J. (2004), 'Deviance information criterion for computing volatility models', Journal of Business and Economic Statistics 22, 107120.

Blanchard, O. J. \& Fisher, S. (1989), Lectures on Macroeconomics, Cambridge University Press.

Bollerslev, T. (1986), 'Generalized autoregressive conditional heterosdasticity', Journal of Econometrics 31, 307-327.

Bollerslev, T. (1990), 'Modeling the coherence in short-run nominal exchange rates: A multivariate generalized arch model.', The Review of Economic and Statistics 72, 498-505.

Bollerslev, T., Chou, R. Y. \& Kroner, K. F. (1992), 'Arch modeling in finance: a review of the theory and empirical evidence', Journal of Econometrics 52, 559.

BOVESPA (2007), Bolsa de valores de são paulo, Technical report, BOVESPA.

Carlin, B. P. \& Louis, T. A. (2000), Bayes and Empirical Bayes methods for data analysis, Chapman and Hall/CRC Press.

Engle, R. (1982), 'Autoregressive conditional heterosdasticity with estimates of the variance of uk inflation.', Econometrica 50, 987-1007.

Engle, R. (2001), 'Garch 101: The use of arch/garch models in applied econometrics', Journal of Economic Perspectives 15, 157-168.

Gelfand, A. E. \& Smith, A. F. M. (1990), 'Sampling-based approaches to calculating marginal densities.', Journal of the American Statistical Association 85, 398-409.

Ghysels, E. (1996), Stochastic volatility, in 'Statistical methods on finance', North-Holland.

Kim, S. \& Shephard, N. (1998), 'Stochastic volatility: likelihood inference and comparison with arch models.', Review of Economic Studies 65, 361-393.

Meyer, R. \& Yu, T. (2000), 'Bugs for a bayesian analysis of stochastic volatility models.', Econometrics Journal 3, 198-215.

Raftery, A. E. (1995), Hypothesis testing and model selection via posterior simulation., in 'Practical Markov Chain Monte Carlo', Chapman and Hall.

Raftery, A. E., Newton, M. A., Satagopan, J. M. \& Krivitsky, P. N. (2006), Estimating the integrated likelihood via posterior simulation using the harmonic mean identity,, Technical report, Center for Statistics and the Social Sciences.

Ross, S. A., Westerfield, R. W. \& Jaffe, J. (2005), Corporate Finance, McGraw Hill Irwin. 
Schwarz, G. (1978), 'Estimating the dimension of a model.', Annals of Statistics 6, 461-464.

Shephard, N. (1996), Statistical aspects of arch and stochastic volatility, in 'Time Series Models in Econometrics', Chapman and Hall.

Smith, A. F. M. \& Roberts, G. O. (1993), 'Bayesian computation the gibbs sampling and related markov chain monte carlo methods', Journal of the Royal Statistical Society Series B 55, 3-23.

Spiegelhalter, D. J., Best, N. G., Carlin, B. P. \& van der Linde, A. (2002), 'Bayesian measures of model complexity and fit', Journal of the Royal Statistical Society Series B 64, 583-639.

Spiegelhalter, D., Thomas, A., Best, N. \& Gilks, W. (1999), 'Winbugs: Bayesian inference using gibbs sampling mrc biostatistics unit'.

Tauchen, G. \& Pitts, M. (1983), 'The price variability-volume relationship on speculative markets', Econometrica 51, 485-505.

Taylor, S. J. (1982), Financial returns modeled by the product of two stochastic processes, a study of daily sugar prices, in 'Time Series Analysis: Theory and Practice', Amsterdam-Holland.

Taylor, S. J. (1994), 'Modelling stochastic volatility: a review and comparative study', Mathematical Finance 4, 183-204. 